\title{
Density of Liquid Lead as Function of Temperature and Pressure Based on the Molecular Dynamics Method
}

\author{
Muhammad Abdul Bashar Imanullah ${ }^{1}$, Artoto Arkundato ${ }^{1, a}$ and Endhah \\ Purwandari $^{1}$ \\ ${ }^{1}$ Physics Department, Faculty of Mathematical and Natural Sciences, Jember, Indonesia \\ aa.arkundato@unej.ac.id
}

\begin{abstract}
Simulation research has been carried out to obtain the formula for mass density of liquid lead as a function of temperature and pressure. The simulation method used is the molecular dynamics method. The potential energy used in the simulation is the Morse potential. From the simulation, it is found that the relationship between the mass density of liquid lead and temperature and pressure can be expressed in the equation $\rho_{P b}=11233-0,9217 \times T$ for pressure $1-5$ atm and $\rho_{P b}=11233-$ $0,9213 \times T$ for pressure $7 \mathrm{~atm}$ in units $\mathrm{kg} / \mathrm{m}^{3}$.
\end{abstract}

Keywords: Liquid Lead, Mass Density, Temperature, Pressure, Molecular Dynamics.

\section{Introduction}

Currently, nuclear power plants have become one of the alternative energy sources which are becoming an interesting choice of many countries. If developed countries have long used nuclear energy to support their heavy industries, developing countries think about the use of this energy in a recent time. The nuclear reactor basically produces heat energy from the process of nuclear fission. The subsequent use in the form of conversion to electricity in large quantities is one of the attractions for the development of this nuclear energy, besides there may concerns for utilization in the field of weaponry.

The development of nuclear reactors for welfare today generally still relies on the design of thermal reactors. There are still many thermal reactors operating in this world. Unfortunately, many thermal reactors are still legacy of old designs. Since the Chernobyl nuclear reactor accident in Ukraine in the past, a safer new concept reactor design is constantly being considered. For this reason currently developing IV generation nuclear reactors. Some of the advantages of this generation IV reactor design are the inherent safety concept.

Liquid metal-cooled fast nuclear reactors, for example liquid lead, are reactor concepts and designs that apply the safety concept inherent in the design so that if there is a potentially catastrophic anomaly in the reactor, the reactor system can automatically shut down the operation of the nuclear reactor without the need for any process of manual shutdown.

More specifically, the liquid lead metal cooled fast nuclear reactor is one of the promising reactor concepts apart from applying the inherent safety concept it is also capable of being made in a modular form and producing high power energy [1]. 
Liquid lead metal and its alloys with other metals such as bismuth are currently promising candidate materials for cooling fast nuclear reactors [2]. There are many advantages compared to cooling water in a slow neutron nuclear reactor. One of them is that it has a high boiling point and has a large thermal conductivity, making it very suitable for cooling in fast reactor designs.

For this reason, in order for the design of a fast nuclear reactor to be made properly, complete nuclear data, for example, information on mass density, is required. Because in the reactor two factors that are very important to note are temperature and pressure, this mass density needs to be known as a function of temperature and pressure.

In this study, therefore, we want to know the density formula as a function of temperature and pressure. To obtain this, the molecular dynamics method will be used so that a lot of data can be obtained for various temperatures and pressures.

\section{Theory}

Molecular dynamics methods in general can be used to predict the physical properties of materials such as melting point, heat conductivity, enthalpy, diffusion coefficient, and so on, including predicting density as a function of temperature. What matters is whether we have sufficient potential energy data to describe the interactions between the atoms of the material system. Arkundato et al has used the molecular dynamics methods to investigate liquid lead coolant [3-4]. In this research, we will use the Morse potential.

In the molecular dynamics method, the Newton motion equation is solved to get the trajectories of all the atoms that make up the material. The relationship between Newton's equations of motion and potential is as follows

$\boldsymbol{F}=m \mathrm{~d}^{2} \boldsymbol{r} / \mathrm{d} t^{2}$

$F=-\mathrm{d} V / \mathrm{d} r$

where $\boldsymbol{F}$ is the interacting force of particles, $\boldsymbol{r}$ position vector of a particle, $\boldsymbol{V}$ is potential energy of interacting particles. The Morse potential for this simulation has a form of [5].

$V(r)=D_{e}\left(1-e^{-a\left(r-r_{e}\right)}\right)^{2}$

where $r_{\mathrm{e}}$ is the equilibrium bond distance from the atom, $D_{\mathrm{e}}$ is the bonding energy balance of an atom and $a$ is a parameter,

$a=\sqrt{\frac{k_{e}}{2 D_{e}}}$

Girifalco dan Weizer (1958) has made table of Morse potential parameters as below [6]. 
Computational and Experimental Research in Materials and Renewable Energy (CERiMRE) Volume 1, Issue 1, page 1-6
Submitted : August 20, 2018

Accepted : October 10, 2018

Online : November 10, 2018

doi : $10.19184 /$ cerimre.v1i1.19541

Table 1. Morse potential parameters of some cubic metals [5]

\begin{tabular}{ccccccc}
\hline Metal & $\alpha a_{0}$ & $B$ & $L(e v) \times 10^{-22}$ & $a=A^{-1}$ & $r_{0}=A$ & $D(e v)$ \\
\hline $\mathrm{Pb}$ & 2.921 & 83.02 & 7.073 & 1.1836 & 3.733 & 0.23480 \\
$\mathrm{Ag}$ & 2.788 & 71.17 & 10.012 & 1.3690 & 3.115 & 0.33230 \\
$\mathrm{Ni}$ & 2.500 & 51.78 & 12.667 & 1.4199 & 2.780 & 0.42050 \\
$\mathrm{Cu}$ & 2.450 & 2.450 & 49.11 & 10.330 & 1.3588 & 0.34290 \\
$\mathrm{Al}$ & 2.347 & 4417 & 8.144 & 1.1646 & 3.253 & 0.27030 \\
$\mathrm{Ca}$ & 2.238 & 39.63 & 4.888 & 0.80535 & 4.569 & 0.16230 \\
$\mathrm{Sr}$ & 2.238 & 39.63 & 4.557 & 0.73776 & 4.988 & 0.15130 \\
$\mathrm{~W}$ & 2.225 & 72.19 & 29.843 & 1.4116 & 3.032 & 0.9906 \\
$\mathrm{Cr}$ & 2.260 & 75.92 & 13.297 & 1.5721 & 2.754 & 0.4414 \\
$\mathrm{Fe}$ & 1.988 & 51.97 & 12.573 & 1.3885 & 2.845 & 0.4174 \\
\hline
\end{tabular}

In our work we will use Morse potential parameter from Girifalco data. Furthermore, based on the trajectory of the atoms of the material as a solution of Newton's equations of motion then using statistical mechanics concepts and theories it can be predicted any physical quantities that we want to know. This physical quantities can be calculated easily when we use the Lammps molecular dynamics software [6] that also we used in this research (https://lammps.sandia.gov/).

\section{Method}

The purpose of this study was to find the mass density relation of liquid lead as a function of temperature and pressure. Physical variables mass density, pressure, temperature were simulated with Lammps software. The procedure for obtaining the relationship between the three variables is carried out according to the following steps:

1. Create a script file that contains data on the position of lead atoms, mass, number of atoms, pressure, temperature, number of integration steps, compute command, etc. Table 2 is a summary of input parameters.

Table 3. Input parameters for Lammps simulation

\begin{tabular}{cc}
\hline Variable & Value \\
\hline Mass of $\mathrm{Pb}$ & 207.2 \\
Lattice constant & $4.950 \AA$ \\
\hline Temperatures & $323 \mathrm{~K}, 423 \mathrm{~K}, 523 \mathrm{~K}, 623 \mathrm{~K}$, \\
& $723 \mathrm{~K}, 823 \mathrm{~K}, 923 \mathrm{~K}, 1023 \mathrm{~K}$ \\
\hline $\mathrm{D}$ & $0.2648 \mathrm{ev}$ \\
$\mathrm{r}_{0}$ & $3.520 \mathrm{~A}$ \\
$a$ & $1.3036 \mathrm{~A}^{-1}$ \\
\hline pressure & $1 \mathrm{~atm}, 5 \mathrm{~atm}, 7 \mathrm{~atm}$ \\
\hline
\end{tabular}

2. Do simulation for different temperature and pressure

3. Make analysis and conclusion

4. Determine mass density as a function of temperature and pressure 


\section{Results}

From lead simulation results for different temperatures and pressures then we can calculated the density as Table 4.

Table 4. Density of liquid lead at various temperatures and pressures

\begin{tabular}{cccc}
\hline Temperature & \multicolumn{3}{c}{ Mass density $\left(\mathrm{kg} / \mathrm{m}^{3}\right)$} \\
\cline { 2 - 4 }$(\mathrm{K})$ & pressure1 atm & pressure 5 atm & pressure 7 atm \\
\hline 323 & 10918.59013 & 10918.71963 & 10918.78437 \\
423 & 10839.89903 & 10840.03266 & 10840.09959 \\
523 & 10758.77752 & 10758.90916 & 10758.97953 \\
623 & 10673.75396 & 10674.02894 & 10674.10180 \\
723 & 10582.58427 & 10582.73939 & 10582.81616 \\
823 & 10479.97194 & 10480.13611 & 10481.66200 \\
923 & 10356.97857 & 10357.16451 & 10357.58981 \\
1023 & 10189.90371 & 10190.05928 & 10190.47230 \\
\hline
\end{tabular}

From Table 4 we can determine the mass density as a function of temperature and pressure using linear regression method,

For pressure 1 atm:

$\rho_{P b}=11233-0,9217 \times T \quad[1 \mathrm{~atm}] \quad[5 \mathrm{~atm}]$

Equation (5) also applies for pressure 5 atm. For pressure 7 atm there is a little different, i.e.,

$\rho_{P b}=11233-0,9213 \times T \quad[7 \mathrm{~atm}]$

Figure 1 shows the mass density of liquid lead at 1 atm as a result of molecular dynamics simulation.

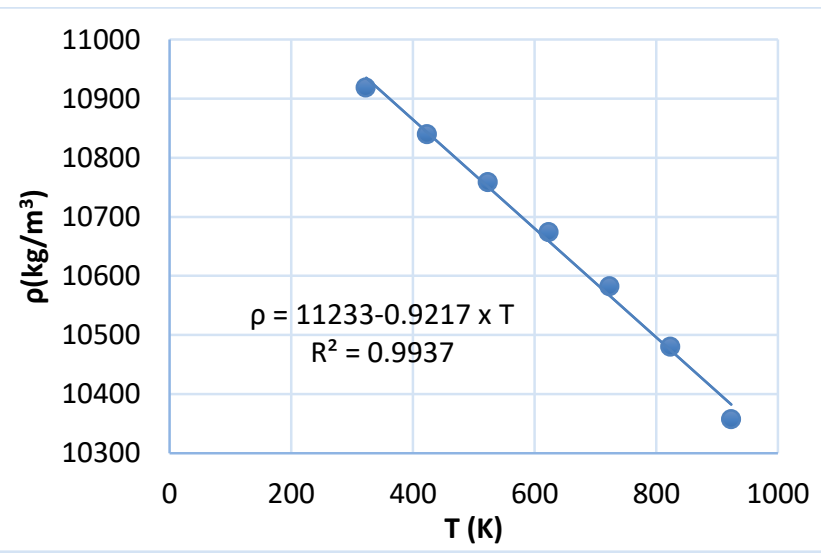

Figure 1. Mass density of liquid lead at $1 \mathrm{~atm}$ 
Let's we compare our simulation result and a reference [7]. Sobelov states that the formulation value for the density of liquid lead at a pressure of 1 atm is:

$\rho_{P b}=11441-1,2795 \times T[1 \mathrm{~atm}]$

We can check the discrepancy between simulation and reference as shown in Table 5.

Table 5. Comparison the mass density by simulation and reference

\begin{tabular}{cccc}
$\begin{array}{c}\text { Temperature } \\
(\mathrm{K})\end{array}$ & $\begin{array}{c}\text { Mass density }\left(\mathrm{kg} / \mathrm{m}^{3}\right) \\
(\text { simulation) }\end{array}$ & $\begin{array}{c}\text { Mass density }\left(\mathrm{kg} / \mathrm{m}^{3}\right) \\
(\text { Sobelov, 2011) }\end{array}$ & $\begin{array}{c}\text { discrepancy } \\
(\%)\end{array}$ \\
\hline 323 & 10935,2909 & 10997,7215 & $0,57 \%$ \\
423 & 10843,1209 & 10869,7715 & $0,25 \%$ \\
523 & 10750,9509 & 10741,8215 & $0,08 \%$ \\
623 & 10658,7809 & 10613,8715 & $0,42 \%$ \\
723 & 10566,6109 & 10485,9215 & $0,77 \%$ \\
823 & 10474,4409 & 10357,9715 & $1,12 \%$ \\
923 & 10382,2709 & 10230,0215 & $1,49 \%$ \\
\hline
\end{tabular}

From Table 5 we can conclude that our simulation results are pretty good when compared to references. In our simulation we have used 500,000 atoms of Pb created by $50 \times 50 \times 50$ boxes of unit cells in FCC crystal structure. Then this also describes that the Morse potential and its parameters is good to formulate the interaction among metallic atoms of lead.

\section{Conclusions}

The simulation results are pretty good when compared to references. The simulation method used is the molecular dynamics method. The potential energy used in the simulation is the Morse potential. In our simulation we have used 500,000 atoms of Pb created by $50 \times 50 \times 50$ boxes of unit cells in FCC crystal structure. Then this also describes that the Morse potential and its parameters is good to formulate the interaction among metallic atoms of lead. From the simulation, it is found that the relationship between the mass density of liquid lead and temperature and pressure can be expressed in the equation $\rho_{P b}=11233-0,9217 \times T$ for pressure $1-5$ atm and $\rho_{P b}=11233-0,9213 \times T$ for pressure 7 atm in units $\mathrm{kg} / \mathrm{m}^{3}$.

\section{References}

[1] T Sofu, 2015, A review of inherent safety characteristics of metal alloy sodium-cooled fast reactor fuel against postulated accidents, Nuclear Engineering and Technology, volume 47, issue 3 , pages 227-239.

[2] J Zhang, N Li, 2008, Review of the Studies on Fundamental Issues in LBE, Journal Nuclear Mater, volume 373, page 351-377.

[3] A Arkundato. Z Su'ud, Abdullah and Widayani, 2013, Molecular Dynamic Simulation on Iron Corrosion Reduction in High Temperature Molten Lead-Bismuth Eutectic, Turk. J. Phys, volume 37, page 132-144. 
[4] A Arkundato, Z Su'ud, M Abdullah, W Sutrisno and M Celino, 2013, Inhibition of iron corrosion in high temperature stagnant liquid lead: A molecular dynamics study, Annals of Nuclear Energy, volume 62, page 298-306.

[5] D D Abajingin, 2012, Solution Of Morse Potential for Face Centre Cube Using Embedded Atom Method, Advance in Physics Theories and Applications, volume 8, page 36-44.

[6] L A Girifalco, V G Weizer, 1959, Application Of The Morse Potential Function To Cubic Metals, Physical Review, volume 114(3), page 687-690.

[7] S Plimpton, 1995, Fast Parallel Algorithms for Short-Range Molecular Dynamics, J Comp Phys, volume 117, page 1-19.

[8] V Sobelov, 2011, Database of Thermophysical Properties of Liquid of Metal Coolats for GEN-IV, Belgium, SKC.CEN. 